\title{
Failure Analysis of a Steel Motorcycle Kickstand
}

\author{
Zainul Huda $\cdot$ Koh Wen Shi $\cdot$ Robert Bulpett
}

Submitted: 1 July 2008/in revised form: 28 April 2009/Published online: 12 May 2009

(c) ASM International 2009

\begin{abstract}
A fractured steel motorcycle kickstand was metallurgically investigated using a range of failure analysis tools [visual examination, energy dispersive X-ray (EDX) analysis, electron microprobe analysis (EPMA), scanning electron microscopy (SEM), fractography, optical microscopy, hardness testing and non-destructive testing (NDT)]. The steel kickstand's composition, its microstructure, electron fractographs, and mechanical test results have been critically interpreted. Some evidence of wear damage, in the failed kickstand, was observed. The microstructural and fractographic analyses showed preexisting micro-cracks which were believed to have grown to result in ductile failure followed by acceleration of corrosion. Recommendations have been made to avoid the failure of the motorcycle kickstand.
\end{abstract}

Keywords Failure analysis - Ductile fracture .

Corrosion $\cdot$ Wears $\cdot$ Electron fractography

\section{Introduction}

This paper reports failure analysis of a kickstand which fractured and failed to function properly causing the motorcycle to collapse to the ground. The failure caused significant damage to the motorcycle and a full investigation

Z. Huda $(\bowtie) \cdot$ K. W. Shi

Department of Mechanical Engineering, University of Malaya, 50603 Kuala Lumpur, Malaysia

e-mail: drzainulhuda@hotmail.com

R. Bulpett

Experimental Techniques Centre, Brunel University of West London, Uxbridge, Middlesex UB8 3PH, UK on the broken kickstand was undertaken to determine the cause(s) of the failure.

There is a continuous and ongoing requirement for improvement in the mechanical properties of materials used in automotive industries [1, 2]. Manufacturers of motorcycles are responsible for making sure that defective parts do not make it into their products. If a motorcycle kickstand is defective, riders may become pinned under a heavy bike or suffer other injuries. Even if the parts are not defective, poor design can lead to similar accidents [3]. For example, a kickstand must be designed and built in such a way as to support the motorcycle and not to lose its effectiveness with repeated use.

A motorcycle kickstand is an accessory component for a motorcycle; it is a swiveling metal bar for holding a twowheeled vehicle, such as a motorcycle, upright when not being ridden. The kickstand is attached to the motorcycle using a bolt and nut, so that its position can be adjusted. In operation it can rotate almost $100^{\circ}$ up and hides under the side cover keeping it safely out of the way of the rider. Figure 1 shows a general view of a kickstand attached to a motorcycle.

\section{Failure Investigations}

In order to investigate the cause(s) of the failure of the kickstand, the following failure analysis techniques and experiments were conducted [4]:

- collection of background data,

- visual inspection,

- electron fractographic examination,

- chemical analysis, and

- metallographic examination. 


\section{Collection of Background Data}

The fracture of the kickstand had occurred on July 11, 2006, giving a service duration of around 18 months during which time the motorcycle was generally exposed to an open air environment with a working temperature in the range between 28 and $30{ }^{\circ} \mathrm{C}$. The fractured motorcycle kickstand was received at an auto-mechanic workshop in Malaysia on August 19, 2006. In collecting service histories of the failed component, special attention was given to environmental details. Complete photographic and written records of the failed component were documented prior to destructive tests. This information was helpful in analyzing the cause(s) of the failure and drawing the conclusions.

\section{Visual Inspection of the Failed Component}

The fractured kickstand, including all its fragments, was subjected to a thorough visual examination before any

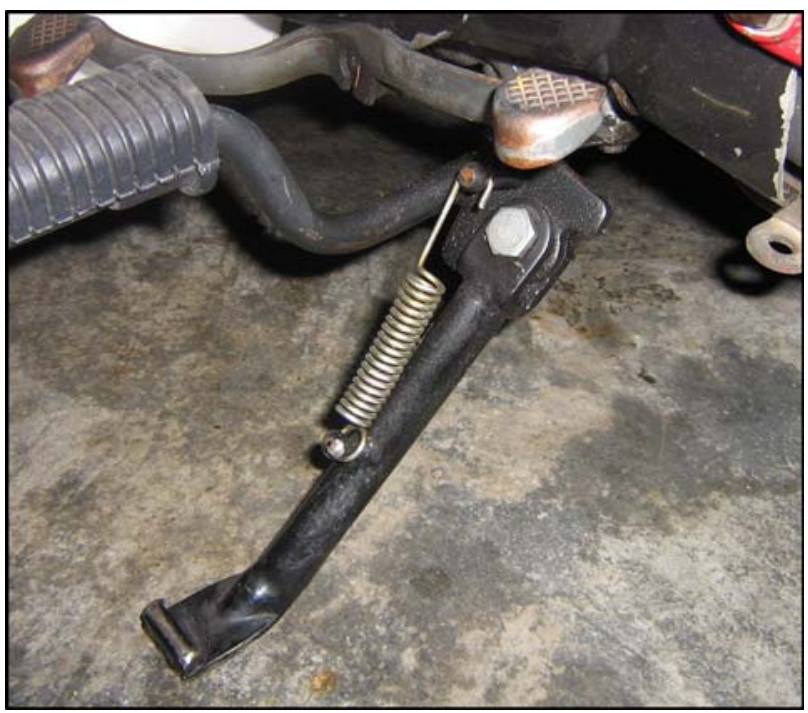

Fig. 1 General view of a kickstand attached to a motorcycle cleaning was undertaken. The recorded dimensions of the kickstand were $26.8 \mathrm{~cm}$ in length, $2.4 \mathrm{~cm}$ in width, and $0.5 \mathrm{~cm}$ in thickness. The external surfaces and the fracture were examined in detail so that failure features such as beach marks, chevron marks, crack of origin and the path of the cracks could be identified. All the physical evidence and features were cataloged and recorded, either in writing or by photographs.

The motorcycle kickstand had broken in the upper portion, as shown in Fig. 2. The fracture occurred at a location approximately $4 \mathrm{~cm}$ from the top-point of the kickstand. The top portion contains a fastener-hole so that the kickstand is firmly attached to the motorcycle frame. The kickstand material surface was protected by black paint; however, in some areas the coating had deteriorated. This is presumably due to exposure to the moist and warm atmosphere.

Unfortunately the fracture surfaces had not been protected immediately after failure and light corrosion, dirt and contaminants were present on the surfaces as shown in the higher magnification images of each half of the fracture (see Fig. 3 and 4). There is also some evidence of wear damage to the area around the bolt hole.

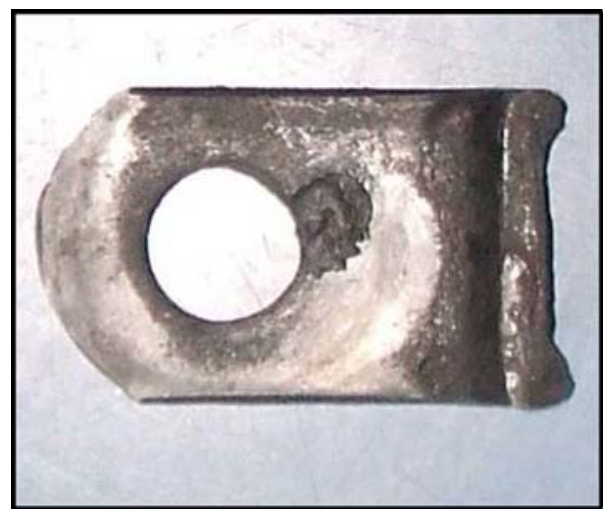

Fig. 3 Enlarged view of fracture surface showing solid dirt and contaminant on the surface
Fig. 2 General view showing the position of fracture (area under red circle indicates the fracture location)

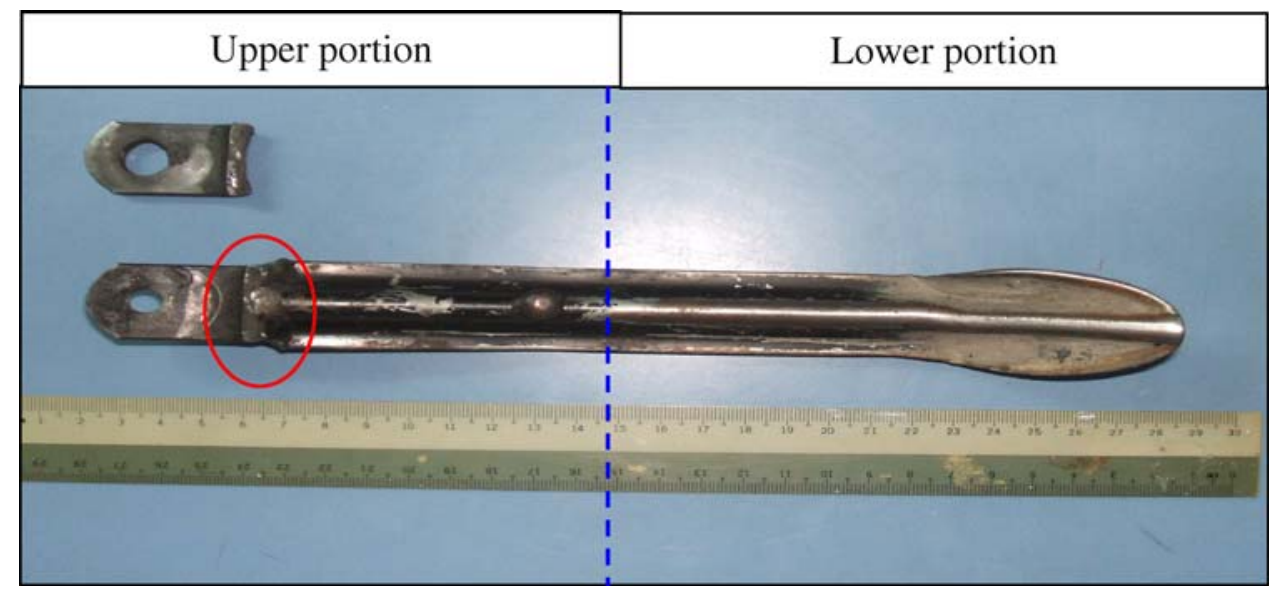


Fig. 4 Enlarged view of fracture surface

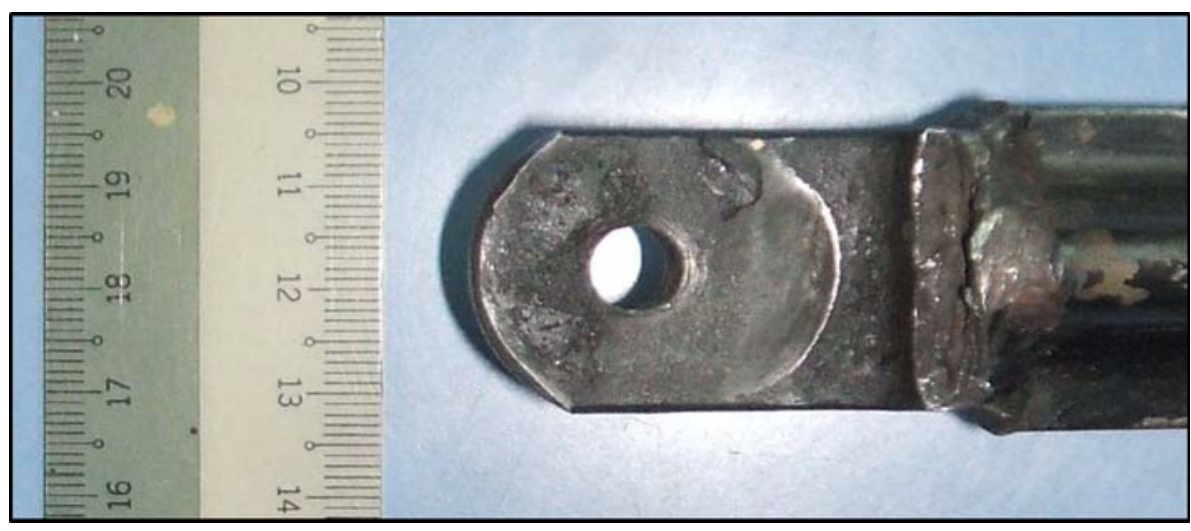

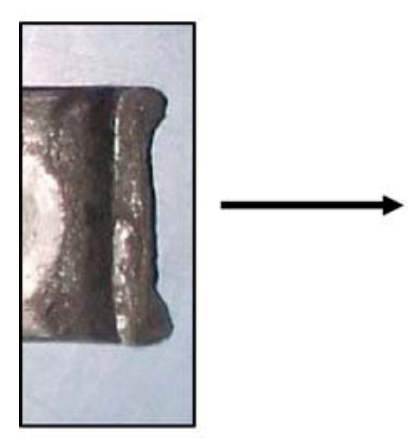

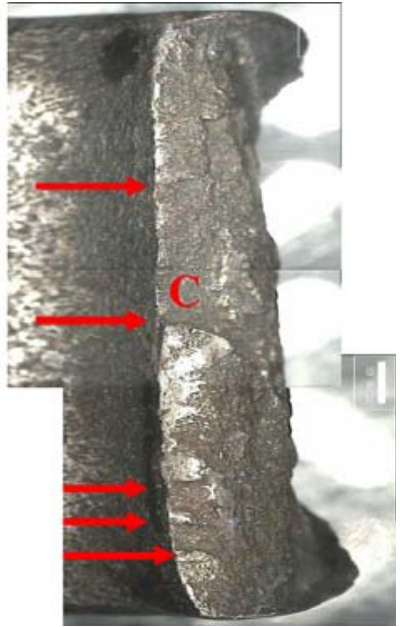

Fig. 5 Fracture surface of kickstand photographed with an optical microscope $(40 \times)$. It shows several crack initiation points (red arrows). $\mathrm{C}=$ significant crack point

The fracture appeared to have initiated at several sites, as shown in Fig. 5. The most significant crack initiation point is indicated as point $\mathrm{C}$. The major and minor crack points were propagating from the inward side to outward side. This would produce a final break at the outer side of the kickstand. This is consistent with the observation that the remaining lug had not been bent during the course of the failure as shown in Fig. 6.

\section{Electron Fractographic Examination}

The fracture surface was examined thoroughly using scanning electron microscopy (SEM) to identify significant failure characteristics. The fracture was initially examined in the 'as-received' condition, but surface detail was obscured by foreign particles or a layer of corrosion or oxidation product, so after initial inspection the specimen was cleaned in an ultrasonic acetone bath. The crack initiation point was observed on the cleaned fracture area, as

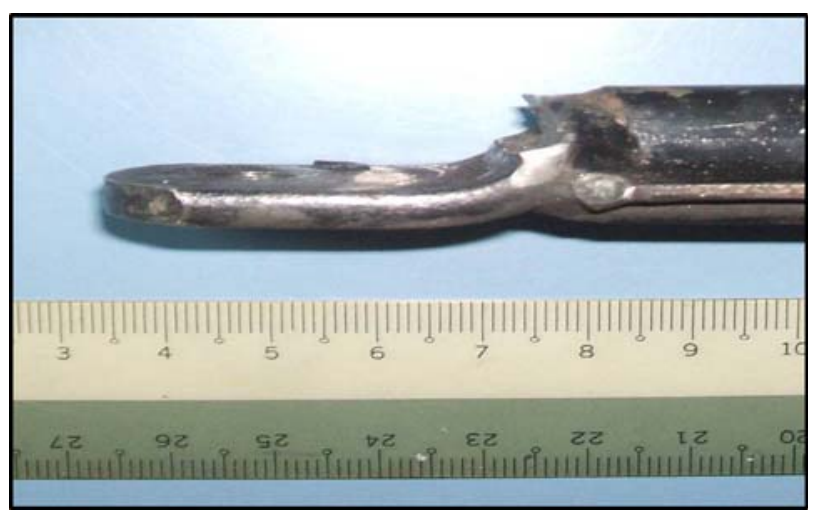

Fig. 6 Side view of the fractured kickstand

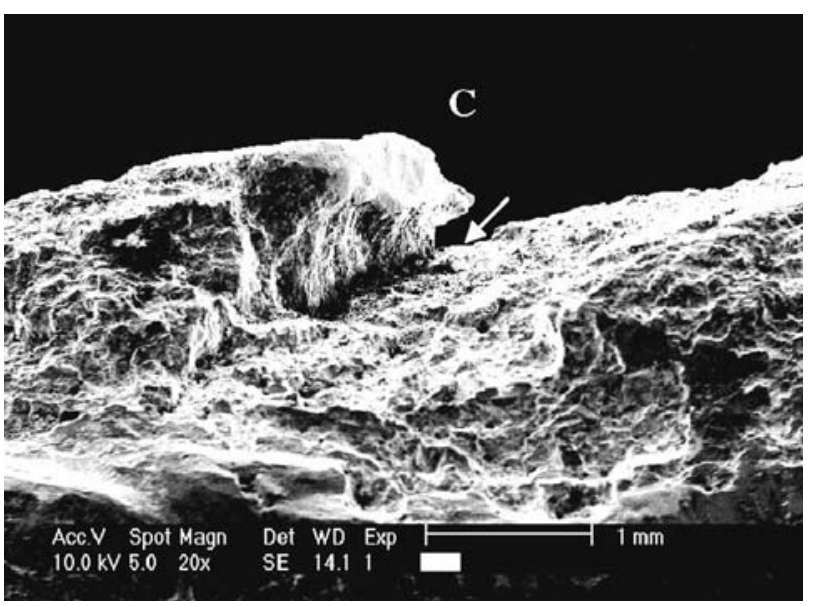

Fig. 7 SEM fractograph showing ductile tearing

shown in Fig. 7. The fracture then propagated by ductile tearing through the thickness of the section. Ductile tearing is a common fracture mechanism in steels [5-7].

The outer surface of this fragment shows some evidence of post-fracture damage with smoothing of the fracture surface and indentations, as shown in Fig. 8. 
Close examination of the fracture initiation site showed evidence of corrosion on the fracture surface and on the adjacent inner surface of the steel lug.

Energy Dispersive Spectroscopy and Electron Probe Microanalysis

Analysis of the brown corrosion product (see Fig. 9) by energy dispersive X-ray spectroscopy (EDS) showed this to be iron oxide with calcium and potassium from contact with soil and a low level of chlorine. It should be noted that

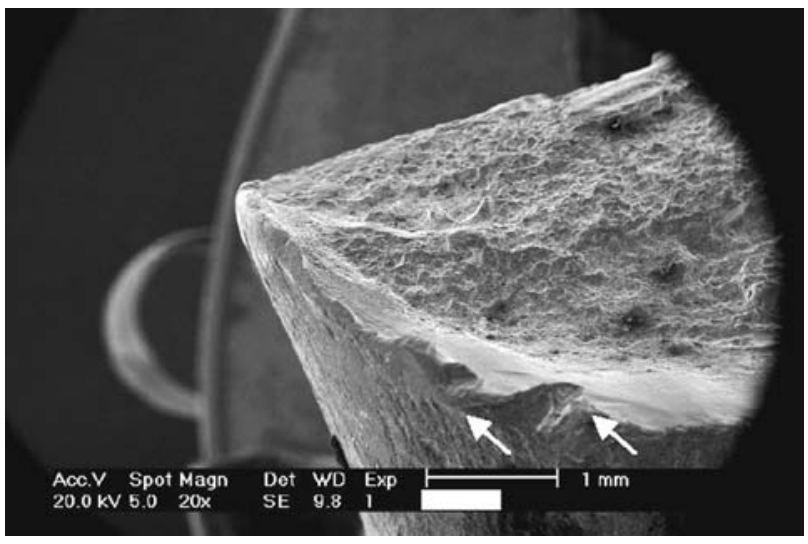

Fig. 8 Damage to edge of fracture and outer surface (indicated by arrows)

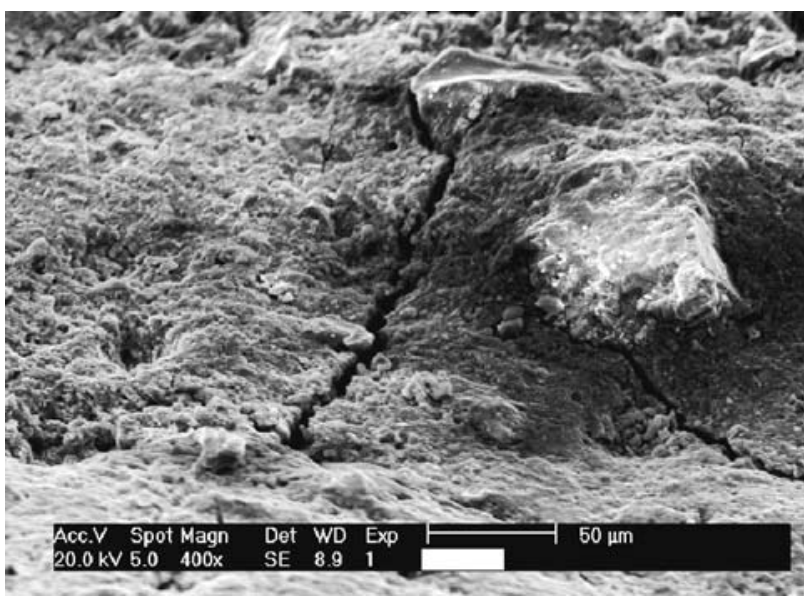

Fig. 9 Cracking in the oxide layer in the failed kickstand even small amounts of chlorine will accelerate corrosion in this type of steel.

A small portion of the kickstand was cut and was subjected to chemical analysis by electron probe microanalysis (EPMA), and was compared to the typical elemental composition of plain carbon steels [8]. The EPMA analysis results are shown in Table 1.

Thus, the chemical composition of the kickstand corresponds to the specification of plain carbon steels. The relatively high content of $\mathrm{Si}$ could be unfavorable and is usually kept below $0.5 \mathrm{wt} . \%$ in plain carbon steels, otherwise it can cause breakdown of the cementite which would result in weakness.

\section{Metallographic Analysis and Hardness Testing}

A longitudinal section through the fracture surface was prepared using standard techniques of grinding, polishing, and etching with a dilute solution of nitric acid in methanol. As we move away from the fracture surface, the microstructure has an equiaxed structure of ferrite grains interspersed with small areas of dark etching pearlite (see Fig. 10).

An interpretation of the microstructure (see Fig. 10) indicates that the material used for kickstand is low-tomedium (plain) carbon steel. This fact can be verified by observing the amounts of ferrite and pearlite present in the microstructure; the carbon content could be estimated in the range of $0.3-0.4 \%$.

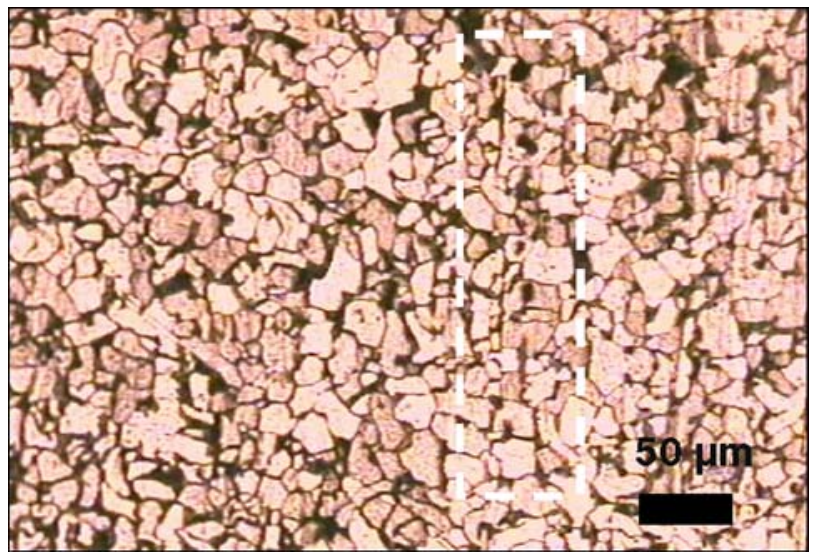

Fig. 10 Microstructure of the steel kickstand

Table 1 Composition of the steel kickstand (wt.\%)

\begin{tabular}{lllllll}
\hline & \multicolumn{1}{c}{$\mathrm{C}$} & $\mathrm{Mn}$ & $\mathrm{P}$ & $\mathrm{Si}$ & $\mathrm{S}$ & $\mathrm{Fe}$ \\
\hline Actual composition, wt.\% & N/A & 0.6 & 0.04 & 0.7 & 0.01 & $\mathrm{Bal}$ \\
Standard composition, wt.\% & $0.2-0.4$ & $1.5 \mathrm{max}$ & 0.05 max & $0.1-0.5$ & $0.05 \mathrm{max}$ & $\mathrm{Bal}$ \\
\hline
\end{tabular}


An average value of hardness measured away from the fracture was measured as $175 \mathrm{HV}$ using a $30 \mathrm{~kg}$ load. This is equivalent to a tensile strength of $570 \mathrm{MPa}$, which is typical for a medium carbon steel in an annealed or normalized condition. Microhardness testing of the region close to the fracture surface using a $50 \mathrm{~g}$ load showed work hardening to $230 \mathrm{HV}$.

\section{Conclusion}

The failure of the kickstand is attributed primarily to fracture by a process of ductile failure following localized corrosion accelerated by the presence of chloride ions. The inner surfaces of the lug showed evidence of wear damage, suggesting that the retaining bolt could have worked loose.

The service environment of the kickstand is an important factor that affects the service life of the kickstand. In this case, the kickstand was exposed to an open environment and this appears to have considerably shortened the service life.

\section{Recommendations}

The use of steel with higher strength and improved corrosion resistance would considerably extend the life of the kickstand. Appropriate heat treatment of medium carbon steel should provide adequate strength whilst corrosion protection can be provided by application of a suitably protective paint finish and lubrication of the moving parts.
The failure appears to have been caused by a combination of inadequate design and material selection in addition to an apparent lack of maintenance resulting in wear and corrosion damage.

Acknowledgments The authors are grateful to the Department of Mechanical Engineering, University of Malaya (UM) to allow Metallurgy Lab and SEM facilities. The permission to use EPMA/SEM granted by Geology Department of UM is also acknowledged.

\section{References}

1. Kabir, M.J., Bulpett, R.: A study of fatigue failure initiation in high cycle high-pressure automotive applications and conventional rotating bending fatigue testing. Int. J. Fatigue 29, 1966-1970 (2007)

2. Bahi, M.A., Lecuyer, P., Fremont, H., Landesman, J.-P.: Sequential environmental stresses tests qualification for automotive components. Microelectron. Reliab. 47, 1680-1684 (2007)

3. Zhang, X., Jin, X., Li, Y., Li, G.: Improved design of the main energy-absorbing automotive parts based on traffic accident analysis. Mater. Des. 29, 403-410 (2008)

4. Colangelo, V.J., Heiser, F.A.: Analysis of Metallurgical Failures. Wiley, New York (1987)

5. Sandvik, A., Østby, E., Thaulow, C.: A probabilistic fracture mechanics model including 3D ductile tearing of bi-axially loaded pipes with surface cracks. Eng. Fract. Mech. 75, 76-96 (2008)

6. Jayadevan, K.R., Berg, E., Thaulow, C., Østby, E., Skallerud, B.: Numerical investigation of ductile tearing in surface cracked pipes using line-springs. Int. J. Solids Struct. 43, 2378-2397 (2006)

7. Moulin, D., Clement, G., Drubay, B., Goudet, G.: Evaluation of ductile tearing in cracked pipes and elbows under bending. Nucl. Eng. Des. 171, 33-43 (1997)

8. Abouei, V., Saghafian, H., Kheirandish, Sh.: Effect of microstructure on the oxidative wear behavior of plain carbon steel. Wear 262, 1225-1231 (2007) 